\title{
Trading Rate for Balanced Queue Lengths for Network Delay Minimization
}

\author{
Jing Yang, Student Member, IEEE and Sennur Ulukus, Member, IEEE
}

\begin{abstract}
We consider a communication channel with two transmitters and one receiver, with an underlying rate region which is approximated as a general pentagon. Different from the Gaussian multiple access channel (MAC) capacity region, the sum-rate on the dominant face of this pentagon is not a constant. We allocate rates from this rate region to users according to their current queue lengths in order to minimize the average delay in the system. We formulate the problem as a Markov decision problem (MDP), and derive the structural properties of the corresponding discounted-cost MDP. We show that the delayoptimal policy has a switch curve structure. For the discountedcost problem, we prove that the switch curve has a limit along one of the dimensions. The delay-optimal policy divides the entire queue state space into two via a switch curve. If the queue state is on one side of the switch curve, the system operates at one of the corner points of the rate pentagon which favors maximum sum-rate. When the queue state switches to the other side of the switch curve, the system operates at the other corner point of the rate pentagon which favors balancing the queue lengths. As a result, the system does not always operate at the sumrate maximizing rate pair, but trades rate for balanced queue lengths for the goal of minimizing the overall delay. The existence of a limit in the switch curve along one of dimensions implies that, once the queue state is beyond the limit, the system always operates at one of the corner points, implying that the queues can be operated partially distributedly.
\end{abstract}

Index Terms -Delay minimization, rate-delay trade-off, queuelength based rate allocation, multiple access channel, Markov decision processes.

\section{INTRODUCTION}

$\mathbf{T}$ RADITIONAL information theory ignores the burstiness of arrivals and the associated issue of delay by assuming that all of the bits have already arrived and are available at the transmitter before the transmission starts. This is necessary to invoke asymptotics (e.g., large block sizes), which is needed to prove reliability of communication. Network and queueing theory, on the other hand, give sophisticated analysis for delay and related issues, but, assume simplified models for the underlying communication rates, which serve as the server rates of the queues. Network theory typically assumes slotted or time-divided communications in order to minimize the

Manuscript received 1 July 2010; revised 17 December 2010. This work was supported by NSF Grants CCF 04-47613, CCF 05-14846, CNS 07-16311, CCF 07-29127, CNS 09-64632 and presented in part at IEEE International Symposium on Information Theory, Austin, TX, June 2010 [1].

J. Yang was with the Department of Electrical and Computer Engineering, University of Maryland, College Park, MD 20742, USA. She is now with the Department of Electrical and Computer Engineering, University of WisconsinMadison, WI 53706, USA (e-mail: yangjing@umd.edu).

S. Ulukus is with the Department of Electrical and Computer Engineering, University of Maryland, College Park, MD 20742, USA (e-mail: ulukus@umd.edu).

Digital Object Identifier 10.1109/JSAC.2011.110509. interactions between the queues, as the analysis of interacting queues is known to be notoriously difficult. Many authors have pointed to the need to bring information and network theory together to jointly address the goals of reliability, high rates and low delay, e.g., [2]. The goal of this paper is to use a general pentagon shaped underlying rate region (hence, nontime-divided transmissions) and determine the optimal rate allocation policy from this available rate region, as a function of the current queue sizes of the users, to minimize the delay.

Reference [3] considers a symmetric Gaussian multiple access channel (MAC), whose capacity region for two-users is a symmetric pentagon. Reference [3] proves that in order to minimize the packet delay, the system should operate at an extreme point of the MAC capacity region, i.e., at one of the two corner points of the symmetric pentagon. In particular, [3] determines explicitly the corner point the system should operate at as a function of the queue sizes, by proving that the larger rate should be given to the user with the larger queue size, hence the name of the proposed policy: longer-queuehigher-rate (LQHR). References [4], [5] extend [3] to fading multiple access channels and prove the delay optimality of the longest-queue-highest-possible-rate (LQHPR) policy in a symmetric scenario. Reference [6] generalizes [3] to a potentially asymmetric setting, and proves that the system should again operate at one of the two corner points of the capacity region, which in this case is a potentially asymmetric pentagon. This proves that the delay-optimal policy has a switch structure, i.e., that the queue state space should be divided into two, and in each region, the system should operate at one of the two corner points. However, unlike the symmetric case in [3], the explicit form of the switch curve is unknown. Reference [7] develops a policy named "modified LQHR" which works at a corner point of the pentagon when the queue lengths are different, and switches to the mid-point of the dominant face of the pentagon when the queue lengths become equal. The "modified LQHR" algorithm is shown to minimize the average bit delay in a symmetric system. The third chapter of [8] extends "modified LQHR" to a symmetric $M$-user scenario. In [9], we consider a discrete-time symmetric Gaussian MAC, and prove that the queue length balancing policy, which minimizes the queue length difference while working on the dominant face of the capacity region in each slot, minimizes the average bit delay in the system.

From the literature above, we observe that the explicit solution of the queue-length based delay-minimization problem is known only for the symmetric Gaussian MAC, where the underlying rate region is a symmetric pentagon. Even for the asymmetric pentagon, the delay-minimizing policy is not 
known. The reason for this is that delay-minimization requires maximizing the throughput at the current time as well as maximizing the throughput in the future. These are often conflicting objectives. The first objective requires maximizing the sum-rate while the second objective requires balancing the queue lengths. Unbalanced queue lengths increases the likelihood of one of the queues becoming empty, which results in inefficiency of transmission, as it decreases the future achievable sum-rates. Thanks to the special properties of the capacity region of the symmetric Gaussian MAC, these two objectives can be achieved simultaneously.

However, having a symmetric pentagon as a capacity region is a peculiarity of the symmetric Gaussian MAC. The capacity region of a general (non-Gaussian) MAC is not a pentagon, it is a union of pentagons [10]. Likewise, the capacity regions of the fading Gaussian MAC [11], the Gaussian MAC with multiple antennas [12], or the Gaussian MAC with user cooperation [13], [14] are not pentagons. In this paper, we will consider a two-user communication channel with a general pentagon rate region. Different from the Gaussian MAC capacity region, the pentagon we assume does not have a $45^{\circ}$ dominant face. The motivation to study such a rate region is two-fold: First, it is the simplest extension of the rate regions studied so far, that changes a characteristic of the rate region in a fundamental way. This characteristic is that the two corner points on the dominant face do not have equal sum-rates. Therefore, in this example rate region, we are able to observe the tension between throughput optimality, i.e., the desire to work at the point that yields the largest sum-rate, and balancing the queue lengths, i.e., the desire to favor the longer queue over the shorter one, more explicitly. Secondly, this asymmetric pentagon with a non- $45^{\circ}$ dominant face can be seen as a crude approximation of a general rate region, as shown in Fig. 1. That is, we can imagine this asymmetric pentagon to be the largest such shape fitting in a general rate region, which may belong to a MAC with fading, multiple antennas, or cooperation.

Our goal in this paper is to assign rate pairs to users from the underlying rate region based on their current queue lengths in order to minimize the average delay in the system. We formulate the problem as a Markov decision problem (MDP) and prove that the delay-optimal policy should operate at one of the two corner points of the rate region. Through value iteration, we prove that a switch curve structure exists in the queue state space. Next, we prove that for the discounted-cost MDP, the switch curve has a limit on one of the queue lengths, i.e., when one of the queue lengths exceeds a threshold, the transmitters always operate at the corner point which has the larger sum-rate (see Fig. 5). That is, the delay-optimal policy favors throughput-optimality (i.e., larger sum-rate) unless the first queue gets close to empty, in which case, the policy favors balancing queue lengths. Our result has two practical implications: First, it gives a partial analytical characterization for the delay-optimal switch curve. Secondly, it implies that we can operate the queues partially distributedly, in that, if the current queue length of the first user is larger than the limit, then this user does not need to know the current queue length of the other user in order to decide about the rate point at which it should operate on the rate region.

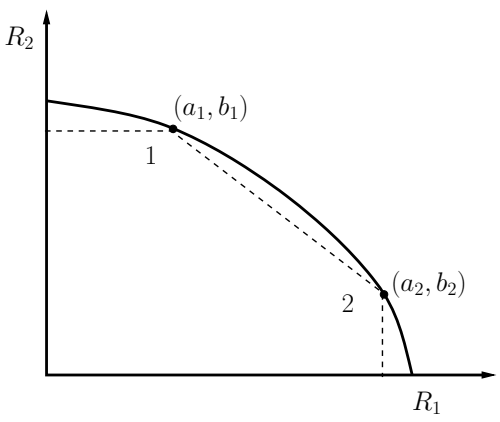

Fig. 1. The asymmetric pentagon rate region with non $-45^{\circ}$ dominant face. Corner point 2 has larger sum-rate, i.e., $a_{2}+b_{2}>a_{1}+b_{1}$.

Finally, we note that, according to the optimal policy, always operating at the maximum sum-rate point is not optimal. With the goal of maximizing the current sum-rate as well as the sum-rate in the future, depending on the current queue lengths, the optimal policy may switch from the maximum sum-rate point to the rate point that favors balancing the queue lengths. This action minimizes the probability that the queues becomes empty in the future, hence maximizes the overall transmission rates, and consequently, minimizes the overall delay. Therefore, we observe that, the optimal rate allocation policy trades some of the instantaneously achievable sumrate in favor of balancing the queue lengths, with the goal of minimizing the overall delay.

\section{System Model AND PROBlem Formulation}

We consider a communication system with two transmitters, and one receiver, as in Fig. 2. The underlying rate region is a general pentagon as shown in Fig. 1. We denote the two corner points of the rate region as points 1 and 2 , with rate pairs $\left(a_{1}, b_{1}\right)$ and $\left(a_{2}, b_{2}\right)$, respectively. Without loss of generality, we assume that $a_{2}+b_{2}>a_{1}+b_{1}$, i.e., that point 2 has a larger sum-rate. We denote the difference between the two sum-rates by $\delta=a_{2}+b_{2}-\left(a_{1}+b_{1}\right)$.

In the medium access control layer, we assume that the packets arrive at the source nodes according to independent Poisson processes with parameters $\lambda_{1}$ and $\lambda_{2}$, see Fig. 2 . We also assume that the packet lengths are independent and identically distributed exponential random variables with unit mean. Therefore, for a given transmission rate $r$, the transmission time for a packet is an exponential random variable with parameter $r$. There is a buffer with infinite capacity at each transmitter, storing the packets until they are transmitted. Let $q_{1}(t), q_{2}(t)$ denote the number of packets in the two buffers at time $t$. The transmitters determine their transmission rates, which are the components of the rate vector $\mathbf{r}$, where $\mathbf{r}$ is in the rate region, based on the current queue length vector $\mathbf{q}(t)=\left(q_{1}(t), q_{2}(t)\right)$. Therefore, on the medium access control layer, the queue lengths evolve according to a continuous-time Markov chain, whose transition rates are determined by the arrival and transmission rates.

According to Little's law [15], minimizing the average delay in the system is equivalent to minimizing the average number of packets in the system. Assuming that the system starts from state $\mathbf{q}(0)$, the delay minimization problem is to obtain an 


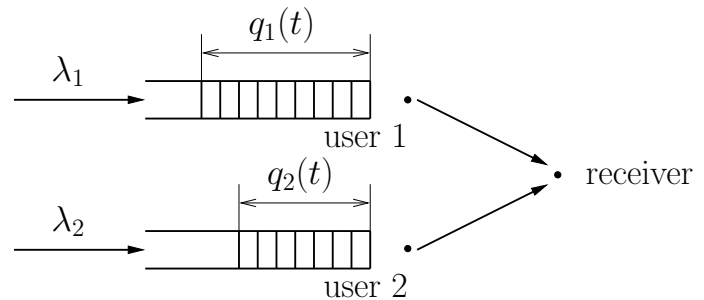

Fig. 2. The system model.

optimal policy, to minimize the long-term average cost:

$$
\limsup _{t \rightarrow \infty} \frac{1}{t} E\left[\int_{0}^{t} \mathbf{q}(s)^{T} \mathbf{e} d s \mid q(0)\right]
$$

where $\mathbf{e}$ is the vector of all ones.

Sampling the system at certain epoches, we can convert the original continuous-time problem into a discrete-time problem [16]. Intuitively, we intend to sample the system at any epoch when an arrival or departure occurs. However, because the transition rates are different at different operating points, the sampling frequency may be different for different states. In order to sample the system at a uniform frequency, we adopt the normalization method in [17]. Since $a_{2}+b_{2}$ is the maximum sum of transmission rates, the maximum total transition rate of the system is $\lambda_{1}+\lambda_{2}+a_{2}+b_{2}$, which we define as $\gamma$. Let us denote the transmission rates of the users as $r_{1}$ and $r_{2}$. If $r_{1}+r_{2}<a_{2}+b_{2}$, we assume that there is a third transmitter transmitting a dummy packet with rate $a_{2}+b_{2}-\left(r_{1}+r_{2}\right)$. Then, we sample at the epoches when either a packet arrives, or a packet (dummy or real) departs. Therefore, the sampling frequency for all of the states will be the same, and the corresponding discrete-time Markov chain will precisely represent the original system.

After sampling and discretizing the continuous-time system, our goal will be to choose $\mathbf{r}$ at every transition epoch to minimize the average delay. Let us denote the indices of the transition epoches as $n, n=1,2, \ldots$. Given the initial queue lengths $\mathbf{q}_{0}$, the delay minimization problem is to determine the optimal policy that minimizes:

$$
\limsup _{N \rightarrow \infty} \frac{1}{N} E\left[\sum_{n=0}^{N-1} \mathbf{q}[n]^{T} \mathbf{e} \mid \mathbf{q}[0]=\mathbf{q}_{0}\right]
$$

Let us define $A_{i}$ and $D_{i}$ to be an arrival or (potential) departure at the $i$ th queue, $i=1,2$. For example, $A_{1} \mathbf{q}=$ $\left(q_{1}+1, q_{2}\right), D_{1} \mathbf{q}=\left(\left(q_{1}-1\right)^{+}, q_{2}\right)$. We first define the corresponding discounted-cost problem with a discount factor $\beta$, and obtain the dynamic programming formulation:

$$
\begin{aligned}
V_{N}^{\beta}(\mathbf{q})= & \mathbf{q}^{T} \mathbf{e}+\beta \gamma^{-1}\left[\lambda_{1} V_{N-1}^{\beta}\left(A_{1} \mathbf{q}\right)+\lambda_{2} V_{N-1}^{\beta}\left(A_{2} \mathbf{q}\right)\right. \\
& +\min _{\mathbf{r} \in C}\left\{r_{1} V_{N-1}^{\beta}\left(D_{1} \mathbf{q}\right)+r_{2} V_{N-1}^{\beta}\left(D_{2} \mathbf{q}\right)\right. \\
& \left.\left.+\left(a_{2}+b_{2}-r_{1}-r_{2}\right) V_{N-1}^{\beta}(\mathbf{q})\right\}\right]
\end{aligned}
$$

where $V_{N}^{\beta}(\mathbf{q})$ is the total discounted cost for the last $N$ stages, $V_{0}^{\beta}(\mathbf{q})=0$, and $C$ is the rate region from which rates $r_{1}$ and $r_{2}$ are chosen. As $N \rightarrow+\infty, V_{N}^{\beta}(\mathbf{q}) \rightarrow V^{\beta}(\mathbf{q})$, which is the unique solution of the optimality equation over finite horizon:

$$
\begin{aligned}
V^{\beta}(\mathbf{q})= & \mathbf{q}^{T} \mathbf{e}+\beta \gamma^{-1}\left[\lambda_{1} V^{\beta}\left(A_{1} \mathbf{q}\right)+\lambda_{2} V^{\beta}\left(A_{2} \mathbf{q}\right)\right. \\
& +\min _{\mathbf{r} \in C}\left\{r_{1} V^{\beta}\left(D_{1} \mathbf{q}\right)+r_{2} V^{\beta}\left(D_{2} \mathbf{q}\right)\right. \\
& \left.\left.+\left(a_{2}+b_{2}-r_{1}-r_{2}\right) V^{\beta}(\mathbf{q})\right\}\right]
\end{aligned}
$$

This is a two-dimensional MDP, which is difficult to solve in general. We first determine some structural properties of the optimal policy.

Lemma $1 V^{\beta}(\mathbf{q})$ is monotonically increasing in $q_{i}, i=1,2$.

Proof: We prove this lemma using induction. First, since $V_{0}^{\beta}(\mathbf{q})=0, V_{N}^{\beta}(\mathbf{q})$ increases monotonically in $q_{1}$ and $q_{2}$ for $N=0$. Then, we assume that this lemma holds for $V_{N}^{\beta}(\mathbf{q}), N>0$, and prove it for $N+1$. Since

$$
\begin{aligned}
V_{N+1}^{\beta}(\mathbf{q})= & \mathbf{q}^{T} \mathbf{e}+\beta \gamma^{-1}\left[\lambda_{1} V_{N}^{\beta}\left(A_{1} \mathbf{q}\right)+\lambda_{2} V_{N}^{\beta}\left(A_{2} \mathbf{q}\right)\right. \\
& +\min _{\mathbf{r} \in C}\left\{r_{1} V_{N}^{\beta}\left(D_{1} \mathbf{q}\right)+r_{2} V_{N}^{\beta}\left(D_{2} \mathbf{q}\right)\right. \\
& \left.\left.+\left(a_{2}+b_{2}-r_{1}-r_{2}\right) V_{N}^{\beta}(\mathbf{q})\right\}\right]
\end{aligned}
$$

Using the assumption that $V_{N}^{\beta}(\mathbf{q})$ is monotonically increasing in $q_{1}$ and $q_{2}$ and the fact that $\mathbf{q}^{T} \mathbf{e}$ is also monotonically increasing in $q_{1}$ and $q_{2}$, in order to prove the monotonicity of $V_{N+1}^{\beta}(\mathbf{q})$ in $q_{1}$ and $q_{2}$, we only need to show that

$\min _{\mathbf{r} \in C}\left\{r_{1} V_{N}^{\beta}\left(D_{1} \mathbf{q}\right)+r_{2} V_{N}^{\beta}\left(D_{2} \mathbf{q}\right)+\left(a_{2}+b_{2}-r_{1}-r_{2}\right) V_{N}^{\beta}(\mathbf{q})\right\}$

is monotonically increasing in $q_{1}$ and $q_{2}$. We compare the values of this expression at two states $A_{1} \mathbf{q}$ and $\mathbf{q}$ as follows

$$
\begin{gathered}
\min _{\mathbf{r} \in C}\left\{r_{1} V_{N}^{\beta}\left(D_{1} A_{1} \mathbf{q}\right)+r_{2} V_{N}^{\beta}\left(D_{2} A_{1} \mathbf{q}\right)\right. \\
\left.+\left(a_{2}+b_{2}-r_{1}-r_{2}\right) V_{N}^{\beta}\left(A_{1} \mathbf{q}\right)\right\} \\
=r_{1}^{*} V_{N}^{\beta}\left(D_{1} A_{1} \mathbf{q}\right)+r_{2}^{*} V_{N}^{\beta}\left(D_{2} A_{1} \mathbf{q}\right) \\
+\left(a_{2}+b_{2}-r_{1}^{*}-r_{2}^{*}\right) V_{N}^{\beta}\left(A_{1} \mathbf{q}\right) \\
\geq r_{1}^{*} V_{N}^{\beta}\left(D_{1} \mathbf{q}\right)+r_{2}^{*} V_{N}^{\beta}\left(D_{2} \mathbf{q}\right) \\
+\left(a_{2}+b_{2}-r_{1}^{*}-r_{2}^{*}\right) V_{N}^{\beta}(\mathbf{q}) \\
\geq \min _{\mathbf{r} \in C}\left\{r_{1} V_{N}^{\beta}\left(D_{1} \mathbf{q}\right)+r_{2} V_{N}^{\beta}\left(D_{2} \mathbf{q}\right)\right. \\
\left.+\left(a_{2}+b_{2}-r_{1}-r_{2}\right) V_{N}^{\beta}(\mathbf{q})\right\}
\end{gathered}
$$

where $\left(r_{1}^{*}, r_{2}^{*}\right)$ minimizes the value of (6) at state $A_{1} \mathbf{q}$. Here the first inequality follows from the assumption that $V_{N}^{\beta}(\mathbf{q})$ is monotonically increasing in $q_{1}$ and $q_{2}$, and the second inequality follows from the fact that $\left(r_{1}^{*}, r_{2}^{*}\right)$ may not be the minimizer of the function in (10).

Comparing (7) and (10), we conclude that the function in (6) is monotonically increasing in $q_{1}$ and $q_{2}$ for $N$. Then, since this is true for any $N$, by taking the limit $V^{\beta}(\mathbf{q})=$ $\lim _{N \rightarrow \infty} V_{N}^{\beta}(\mathbf{q})$ is monotonically increasing in $q_{1}$ and $q_{2}$. 
Lemma 2 The optimal operating point must lie on the boundary of the rate region. In addition, it must be one of the two corner points.

Proof: The first half of Lemma 2 can be proved using Lemma 1. If the optimal operating point $\left(r_{1}, r_{2}\right)$ is not on the boundary but is in the interior of the rate region, then, we can always find another operating point $\left(r_{1}^{\prime}, r_{2}^{\prime}\right)$ on the boundary, where $r_{1}^{\prime}=\alpha r_{1}, r_{2}^{\prime}=\alpha r_{2}$, and $\alpha>1$. Then,

$$
\begin{aligned}
r_{1}^{\prime} & V_{N-1}^{\beta}\left(D_{1} \mathbf{q}\right)+r_{2}^{\prime} V_{N-1}^{\beta}\left(D_{2} \mathbf{q}\right) \\
& +\left(a_{2}+b_{2}-r_{1}^{\prime}-r_{2}^{\prime}\right) V_{N-1}^{\beta}(\mathbf{q}) \\
= & \left(a_{2}+b_{2}\right) V_{N-1}^{\beta}(\mathbf{q})+\alpha r_{1}\left(V_{N-1}^{\beta}\left(D_{1} \mathbf{q}\right)-V_{N-1}^{\beta}(\mathbf{q})\right) \\
& +\alpha r_{2}\left(V_{N-1}^{\beta}\left(D_{2} \mathbf{q}\right)-V_{N-1}^{\beta}(\mathbf{q})\right) \\
\leq & \left(a_{2}+b_{2}\right) V_{N-1}^{\beta}(\mathbf{q})+r_{1}\left(V_{N-1}^{\beta}\left(D_{1} \mathbf{q}\right)-V_{N-1}^{\beta}(\mathbf{q})\right) \\
& +r_{2}\left(V_{N-1}^{\beta}\left(D_{2} \mathbf{q}\right)-V_{N-1}^{\beta}(\mathbf{q})\right) \\
= & r_{1} V_{N-1}^{\beta}\left(D_{1} \mathbf{q}\right)+r_{2} V_{N-1}^{\beta}\left(D_{2} \mathbf{q}\right) \\
& +\left(a_{2}+b_{2}-r_{1}-r_{2}\right) V_{N-1}^{\beta}(\mathbf{q})
\end{aligned}
$$

where the inequality follows from Lemma 1 , and the fact that $\alpha>1$. This contradicts with the optimality of $\left(r_{1}, r_{2}\right)$. Thus, the optimal operating point must lie on the boundary of the rate region. Therefore, we only need to focus on the dominant face of the capacity region. Any point $\left(r_{1}, r_{2}\right)$ on the dominant face can be expressed as a linear combination of the two corner points. Thus, we have

$$
\begin{aligned}
\min _{\mathbf{r} \in C} & \left\{r_{1} V_{N-1}^{\beta}\left(D_{1} \mathbf{q}\right)+r_{2} V_{N-1}^{\beta}\left(D_{2} \mathbf{q}\right)\right. \\
& \left.+\left(a_{2}+b_{2}-r_{1}-r_{2}\right) V_{N-1}^{\beta}(\mathbf{q})\right\} \\
= & \min _{\rho \in(0,1)}\left\{\rho\left(a_{1} V_{N-1}^{\beta}\left(D_{1} \mathbf{q}\right)+b_{1} V_{N-1}^{\beta}\left(D_{2} \mathbf{q}\right)+\delta V_{N-1}^{\beta}(\mathbf{q})\right)\right. \\
& \left.+(1-\rho)\left(a_{2} V_{N-1}^{\beta}\left(D_{1} \mathbf{q}\right)+b_{2} V_{N-1}^{\beta}\left(D_{2} \mathbf{q}\right)\right)\right\} \\
= & a_{2} V_{N-1}^{\beta}\left(D_{1} \mathbf{q}\right)+b_{2} V_{N-1}^{\beta}\left(D_{2} \mathbf{q}\right)+\min \left\{0, \delta V_{N-1}^{\beta}(\mathbf{q})\right. \\
& \left.+\left(b_{1}-b_{2}\right) V_{N-1}^{\beta}\left(D_{2} \mathbf{q}\right)+\left(a_{1}-a_{2}\right) V_{N-1}^{\beta}\left(D_{1} \mathbf{q}\right)\right\}
\end{aligned}
$$

where the last equality follows from the fact that the minimizer for a linear function must be one of the end points.

Let $T$ be an operator defined on real-valued functions as:

$$
\begin{aligned}
T f(\mathbf{q})= & \mathbf{q}^{T} \mathbf{e}+\beta \gamma^{-1}\left[\lambda_{1} f\left(A_{1} \mathbf{q}\right)+\lambda_{2} f\left(A_{2} \mathbf{q}\right)\right. \\
& +a_{2} V_{N-1}^{\beta}\left(D_{1} \mathbf{q}\right)+b_{2} V_{N-1}^{\beta}\left(D_{2} \mathbf{q}\right)+\min \{0 \\
& \left.\left.\left(a_{1}-a_{2}\right) f\left(D_{1} \mathbf{q}\right)+\left(b_{1}-b_{2}\right) f\left(D_{2} \mathbf{q}\right)+\delta f(\mathbf{q})\right\}\right]
\end{aligned}
$$

Therefore, the dynamic programming optimality equation can be written as

$$
V_{N+1}^{\beta}(\mathbf{q})=T V_{N}^{\beta}(\mathbf{q})
$$

\section{An Inductive Proof of the Switch Structure}

In this section, we prove that the delay-optimal policy has a switch structure. In order to prove that, we first define a set of functions with properties which are sufficient to have a switch structure. We show that these properties are preserved under the operator $T$. Since $V_{0}^{\beta}=0$ is within this set, using induction, we will show that $V^{\beta}$ will be within this set.

Let us define $\mathcal{F}$ to be the set of real-valued functions such that:

1) $f(\mathbf{q})$ is increasing in $q_{1}$ and $q_{2}$.

2) $f(\mathbf{q}+\mathbf{x})-f(\mathbf{q})$ is increasing in $q_{1}$ and $q_{2}$ for any fixed $\mathrm{x}$.

3) $\left(a_{1}-a_{2}\right) f\left(D_{1} \mathbf{q}\right)+\left(b_{1}-b_{2}\right) f\left(D_{2} \mathbf{q}\right)+\delta f(\mathbf{q})$ is increasing in $q_{1}$.

4) $\left(a_{1}-a_{2}\right) f\left(D_{1} \mathbf{q}\right)+\left(b_{1}-b_{2}\right) f\left(D_{2} \mathbf{q}\right)+\delta f(\mathbf{q})$ is decreasing in $q_{2}$.

Then, we have the following lemma.

Lemma 3 If $f \in \mathcal{F}$, then $T f \in \mathcal{F}$.

The proof of Lemma 3, when $\delta=0$, can be found in [18]. When $\delta \neq 0$, the proof is different, and is provided in Appendix A.

Lemma $4 V_{n}^{\beta}(\mathbf{q}) \in \mathcal{F}$ for all $n$.

This lemma can be verified as follows. Since $V_{0}^{\beta}=0, V_{0}^{\beta}$ is in $\mathcal{F}$. Using Lemma 3 recursively, we have $V_{n}^{\beta}(\mathbf{q}) \in \mathcal{F}$ for $n=0,1,2, \ldots$.

We now define the switch function:

$$
\begin{gathered}
s_{n}\left(q_{1}\right)=\min \left\{q_{2}:\left(a_{1}-a_{2}\right) V_{n}^{\beta}\left(D_{1} \mathbf{q}\right)+\left(b_{1}-b_{2}\right) V_{n}^{\beta}\left(D_{2} \mathbf{q}\right)\right. \\
\left.+\delta V_{n}^{\beta}(\mathbf{q}) \leq 0\right\}
\end{gathered}
$$

A generic switch function is shown in Fig. 3. As we state in the following theorem, the optimal rate assignment problem has a switch structure.

Theorem 1 The optimal policy for the discounted-cost MDP has a switch structure, i.e., $s_{n}\left(q_{1}\right)$ is increasing for every $n$.

This theorem can be proved using properties 3) and 4) of $V_{n}^{\beta}(\mathbf{q})$. The switch curve partitions the queue state space into two parts, each corresponding to one of the two operating points (corner points of the pentagon). Following the arguments in [18], [6], we can prove that the switch structure still exists when $\beta \rightarrow 1$, i.e., for the average cost problem.

While we have proved that the optimal policy has a switch structure, i.e., that the queue state space is divided into two, where in each region the optimal policy operates the system at one of the two corner points, a closed form solution for this switch curve is not known in general. The switch curve is explicitly known only for one special case, which is the symmetric Gaussian MAC case, where the rate region is a symmetric pentagon with a $45^{\circ}$ dominant face. In that case the switch curve is a $45^{\circ}$ straight line emanating from the origin, i.e., $s_{n}\left(q_{1}\right)=q_{1}$, as shown in Fig. 4. This implies that the system operates at one of the corner points when $q_{1}>q_{2}$, and at the other corner point when $q_{1}<q_{2}$. This results in the LQHR policy in [3]. In the asymmetric Gaussian MAC case, where the rate region is an asymmetric pentagon, but with still a $45^{\circ}$ dominant face, even though it is known that a switch curve structure exists, the explicit form of the switch curve is 


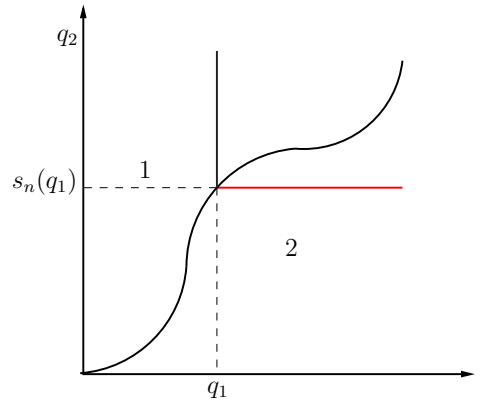

Fig. 3. The switch structure of the optimal policy.

not known [6]. In the next section, we will show that, in this more general case where we have an asymmetric pentagon rate region with a non- $45^{\circ}$ dominant face, even though we do not have an explicit formula for the switch curve, we show that we have a limit on the switch curve along one of the dimensions.

\section{The Limit ON ThE Switch CURVE}

Although we have shown that the delay optimal policy has a switch structure, it is difficult to obtain the exact switch curve analytically. In this section, we will show that the switch curve is bounded in the $q_{1}$-dimension. In other words, we can find a threshold $N$, such that, for all $q_{1}$ greater than this threshold, the optimal operating point is the second corner point of the pentagon. In order to prove this, we start from an initial function $f_{0}$, which is linear in $q_{1}+q_{2}$. We will use $f_{0}$ to approximate $V^{\beta}$ over a large portion of the state space. Specifically, this region includes states q with $q_{1}, q_{2}>N$, where $N$ is a large enough number. Let us define:

$$
f_{0}(\mathbf{q})=\frac{1}{1-\beta}\left(q_{1}+q_{2}\right)+\frac{\beta}{(1-\beta)^{2}} \frac{\lambda_{1}+\lambda_{2}-a_{2}-b_{2}}{\lambda_{1}+\lambda_{2}+a_{2}+b_{2}}
$$

Clearly, $f_{0} \in \mathcal{F}$. It is easy to verify that

$$
T f_{0}(\mathbf{q})-f_{0}(\mathbf{q})= \begin{cases}0 & q_{1}, q_{2} \neq 0 \\ \frac{\beta\left(a_{2}+b_{2}\right)}{\gamma(1-\beta)} & \mathbf{q}=\mathbf{0} \\ \frac{\beta\left(1 a^{+} \delta\right)}{\gamma(1-\beta)} & q_{1}=0 \\ \frac{\beta b_{2}}{\gamma(1-\beta)} & q_{2}=0\end{cases}
$$

that is, $T f_{0}$ and $f_{0}$ differ only on the boundary, and for all states away from the boundary, these two functions have the same value. This is a key property that will be essential in this section. Note that under the operator $T$, the difference caused by the boundary only propagates into the interior region of the state space by one layer in each iteration; rest of the states are not affected by the operator.

Let us define:

$$
|f|_{k}=\max \left\{f(\mathbf{q}): q_{1}, q_{2} \geq 0, q_{1}+q_{2} \leq k\right\}
$$

which is the maximum value of the function $f$ in the region where the sum of the queue lengths is less than $k$. Similarly, let us define

$$
|f|_{\infty}=\sup \left\{f(\mathbf{q}): q_{1}, q_{2} \geq 0\right\}
$$

which is allowed to be infinity. Then, we have the following property.

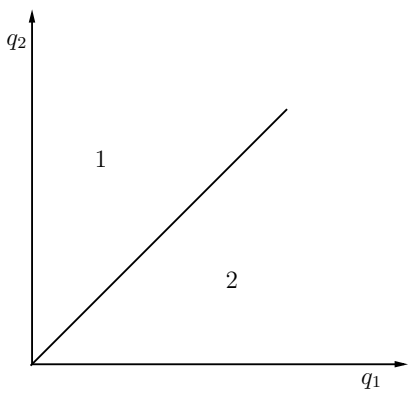

Fig. 4. The switch structure for a symmetric Gaussian MAC.

Lemma 5 For $\forall f, g \in \mathcal{F},|T f-T g|_{k} \leq \beta|f-g|_{k+1}$.

\section{Proof:}

$$
\begin{aligned}
& T f(\mathbf{q})-T g(\mathbf{q}) \\
& =\beta \gamma^{-1}\left[\lambda_{1} f\left(A_{1} \mathbf{q}\right)+\lambda_{2} f\left(A_{2} \mathbf{q}\right)-\lambda_{1} g\left(A_{1} \mathbf{q}\right)-\lambda_{2} g\left(A_{2} \mathbf{q}\right)\right. \\
& +\min \left\{a_{1} f\left(D_{1} \mathbf{q}\right)+b_{1} f\left(D_{2} \mathbf{q}\right)+\delta f(\mathbf{q}), a_{2} f\left(D_{1} \mathbf{q}\right)+b_{2} f\left(D_{2} \mathbf{q}\right)\right\} \\
& \left.-\min \left\{a_{1} g\left(D_{1} \mathbf{q}\right)+b_{1} g\left(D_{2} \mathbf{q}\right)+\delta g(\mathbf{q}), a_{2} g\left(D_{1} \mathbf{q}\right)+b_{2} g\left(D_{2} \mathbf{q}\right)\right\}\right]
\end{aligned}
$$

Since $|\min \{a, b\}-\min \{c, d\}| \leq \max \{|a-c|,|b-d|\}$, we have

$$
\begin{aligned}
& \quad|T f-T g|_{k} \\
& \leq \beta \gamma^{-1}\left[\lambda_{1}|f-g|_{k+1}+\lambda_{2}|f-g|_{k+1}\right. \\
& \quad+\max \left\{a_{1}|f-g|_{k-1}+b_{1}|f-g|_{k-1}+\delta|f-g|_{k}\right. \\
& \left.\left.\quad \quad a_{2}|f-g|_{k-1}+b_{2}|f-g|_{k-1}\right\}\right] \\
& \leq \beta \gamma^{-1}\left(\lambda_{1}+\lambda_{2}+a_{2}+b_{2}\right)|f-g|_{k+1} \\
& =\beta|f-g|_{k+1}
\end{aligned}
$$

completing the proof.

Lemma $6 T^{n} f_{0}$ converges to a function $f$ as $n \rightarrow+\infty$, and $T f=f$.

Proof: Since $f_{0} \in \mathcal{F}, T^{n} f_{0} \in \mathcal{F}$ for any $n>0$.

$$
\begin{aligned}
\left|T^{n+1} f_{0}-T^{n} f_{0}\right|_{k} & \leq \beta\left|T^{n} f_{0}-T^{n-1} f_{0}\right|_{k+1} \\
& \leq \beta^{n}\left|T f_{0}-f_{0}\right|_{k+n} \\
& \leq \frac{\beta^{n+1}\left(a_{2}+b_{2}\right)}{\gamma(1-\beta)}
\end{aligned}
$$

where (28) follows from (20). We observe that (28) does not depend on $k$, thus, $\left|T^{n+1} f_{0}-T^{n} f_{0}\right|_{\infty}$ is uniformly bounded by (28). Since $\beta<1$, the right hand side of (28) forms a Cauchy sequence, therefore, $T^{n} f_{0}$ converges to a function $f$ pointwise. In other words, for any $\epsilon$, we can find an $N_{1}(\epsilon)$ such that when $n \geq N_{1}(\epsilon)$, we have $\left|f-T^{n-1} f_{0}\right|_{\infty} \leq \epsilon$. Thus, for such $n$, we have

$$
\begin{aligned}
|T f-f|_{\infty} & \leq\left|T f-T^{n} f_{0}\right|_{\infty}+\left|T^{n} f_{0}-f\right|_{\infty} \\
& \leq \beta\left|f-T^{n-1} f_{0}\right|_{\infty}+\left|T^{n} f_{0}-f\right|_{\infty} \\
& \leq(\beta+1) \epsilon=\epsilon^{\prime}
\end{aligned}
$$


Therefore, for any $\epsilon^{\prime}$, we can find a $n>N_{1}\left(\frac{\epsilon^{\prime}}{\beta+1}\right)$, such that $|T f-f|_{\infty} \leq \epsilon^{\prime}$. In other words, $T f$ and $f$ are arbitrarily close. Thus, $T f=f$.

Lemma 7 Let $V_{0}^{\beta}(\mathbf{q})=0$, then, $V_{n}^{\beta}(\mathbf{q})=T^{n} V_{0}^{\beta}(\mathbf{q})$ converges to $V^{\beta}(\mathbf{q})$, and $f(\mathbf{q})=V^{\beta}(\mathbf{q})$.

Proof: In order to prove that $f(\mathbf{q})=V^{\beta}(\mathbf{q})$ pointwise, we start from the following:

$$
\begin{aligned}
& \left|f-V^{\beta}\right|_{k} \\
& \leq\left|f-T^{n} f_{0}\right|_{k}+\left|T^{n} f_{0}-V_{n}^{\beta}\right|_{k}+\left|V_{n}^{\beta}-V^{\beta}\right|_{k} \\
& \leq\left|f-T^{n} f_{0}\right|_{k}+\beta\left|T^{n-1} f_{0}-V_{n-1}^{\beta}\right|_{k+1}+\left|V_{n}^{\beta}-V^{\beta}\right|_{k} \\
& \leq\left|f-T^{n} f_{0}\right|_{k}+\left|V_{n}^{\beta}-V^{\beta}\right|_{k}+\beta^{n}\left|f_{0}-V_{0}^{\beta}\right|_{k+n} \\
& =\left|f-T^{n} f_{0}\right|_{k}+\left|V_{n}^{\beta}-V^{\beta}\right|_{k} \\
& \quad+\beta^{n}\left(\frac{n+k}{1-\beta}+\frac{\beta}{(1-\beta)^{2}} \frac{\lambda_{1}+\lambda_{2}-a_{2}-b_{2}}{\lambda_{1}+\lambda_{2}+a_{2}+b_{2}}\right) \\
& \leq \epsilon_{1}+\epsilon_{2}+\epsilon_{3}
\end{aligned}
$$

where (33) follows from Lemma 5, (35) follows from the definition of $f_{0}$, and (36) follows from the fact that $T^{n} f_{0}$ converges to $f_{0}, V_{n}^{\beta}$ converges to $V^{\beta}$, and $\beta^{n} n \rightarrow 0$. Therefore, when $n$ is large enough, we have the difference bounded by (36). We note that (36) does not depend on $k$, thus $f(\mathbf{q})=V^{\beta}(\mathbf{q})$ for any point $\mathbf{q}$.

Lemma 5 means that starting from $f_{0}$ and performing the iterations, $V^{\beta}$ converges to the same function if we started from $V_{0}^{\beta}=0$. The convergence point is the unique solution of the optimality equation (4). Next, we will prove that $f(\mathbf{q})$ gets arbitrarily close to $f_{0}(\mathbf{q})$ when $q_{1}, q_{2} \rightarrow+\infty$.

Lemma $8\left|f-T^{n} f_{0}\right|_{\infty} \leq \frac{\beta^{n+1}\left(a_{2}+b_{2}\right)}{\gamma(1-\beta)^{2}}$.

\section{Proof:}

$$
\begin{aligned}
\mid & T^{n+p} f_{0}-\left.T^{n} f_{0}\right|_{k} \\
\leq & \left|T^{n+p} f_{0}-T^{n+p-1} f_{0}\right|_{k}+\left|T^{n+p-1} f_{0}-T^{n+p-2} f_{0}\right|_{k} \\
& +\cdots+\left|T^{n+1} f_{0}-T^{n} f_{0}\right|_{k} \\
\leq & \left(\beta^{n+p-1}+\beta^{n+p-2}+\cdots+\beta^{n}\right)\left|T f_{0}-f_{0}\right|_{k+n+p} \\
\leq & \frac{\beta^{n}\left(1-\beta^{p}\right)}{1-\beta} \frac{\beta\left(a_{2}+b_{2}\right)}{\gamma(1-\beta)}
\end{aligned}
$$

Note that (39) does not depend on $k$, therefore, $\mid T^{n+p} f_{0}-$ $\left.T^{n} f_{0}\right|_{\infty}$ is uniformly bounded, and we have

$$
\begin{aligned}
\left|f-T^{n} f_{0}\right|_{\infty} & =\lim _{p \rightarrow \infty}\left|T^{n+p} f_{0}-T^{n} f_{0}\right|_{\infty} \\
& =\frac{\beta^{n+1}\left(a_{2}+b_{2}\right)}{\gamma(1-\beta)^{2}}
\end{aligned}
$$

Theorem $2 f(\mathbf{q})$ gets arbitrarily close to $f_{0}(\mathbf{q})$ when $q_{1}, q_{2} \rightarrow+\infty$. Therefore, the switch curve has a limit on $q_{1}$.

Proof: For any fixed state q, we have

$$
\left|f(\mathbf{q})-f_{0}(\mathbf{q})\right| \leq\left|f(\mathbf{q})-T^{n} f_{0}(\mathbf{q})\right|+\left|T^{n} f_{0}(\mathbf{q})-f_{0}(\mathbf{q})\right|
$$

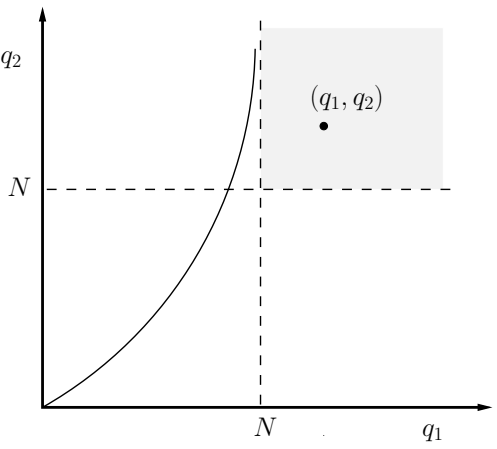

Fig. 5. The switch curve of the discounted-cost MDP.

Based on Lemma 8, we can see that for $\forall \epsilon$, there exists $N(\epsilon)$, such that $\left|f-T^{N(\epsilon)} f_{0}\right|_{\infty} \leq \epsilon$. From the definition in (22),

$$
\left|f(\mathbf{q})-T^{N(\epsilon)} f_{0}(\mathbf{q})\right| \leq\left|f-T^{N(\epsilon)} f_{0}\right|_{\infty} \leq \epsilon
$$

At the same time, from (20), we know that $T^{N(\epsilon)} f_{0}(\mathbf{q})$ only differs from $f_{0}(\mathbf{q})$ over the states which are within $N(\epsilon)$ layers away from the boundary. Thus, for all $q_{1}>N(\epsilon), q_{2}>N(\epsilon)$,

$$
T^{N(\epsilon)} f_{0}(\mathbf{q})-f_{0}(\mathbf{q})=0
$$

Therefore, combining (42)-(44), for any q, $q_{1}>N(\epsilon), q_{2}>$ $N(\epsilon),(42)$ is bounded by

$$
\left|f(\mathbf{q})-f_{0}(\mathbf{q})\right| \leq\left|f-f_{0}\right|_{\infty}+0=\epsilon
$$

i.e., $-\epsilon \leq f(\mathbf{q})-f_{0}(\mathbf{q}) \leq \epsilon$. Thus, in this region, as shown in Fig. 5, we have

$$
\begin{array}{rl}
a_{1} & f\left(D_{1} \mathbf{q}\right)+b_{1} f\left(D_{2} \mathbf{q}\right)+\delta f(\mathbf{q})-a_{2} f\left(D_{1} \mathbf{q}\right)-b_{2} f\left(D_{2} \mathbf{q}\right) \\
= & \left(b_{1}-b_{2}\right) f\left(D_{2} \mathbf{q}\right)+\delta f(\mathbf{q})-\left(a_{2}-a_{1}\right) f\left(D_{1} \mathbf{q}\right) \\
\geq & \left(b_{1}-b_{2}\right)\left(f_{0}\left(D_{2} \mathbf{q}\right)-\epsilon\right)+\delta\left(f_{0}(\mathbf{q})-\epsilon\right) \\
& -\left(a_{2}-a_{1}\right)\left(f_{0}\left(D_{1} \mathbf{q}\right)+\epsilon\right) \\
= & \frac{\delta}{1-\beta}-2\left(a_{2}-a_{1}\right) \epsilon
\end{array}
$$

where the inequality follows from (45) and (48) follows from the definition of $f_{0}$ in (19). Therefore, when

$$
\epsilon \leq \frac{\delta}{2\left(a_{2}-a_{1}\right)(1-\beta)}
$$

(48) is always greater than zero, thus point 2 is always better than point 1 . From Lemma 8, let

$$
\epsilon=\frac{\beta^{n+1}\left(a_{2}+b_{2}\right)}{\gamma(1-\beta)^{2}}=\frac{\delta}{2\left(a_{2}-a_{1}\right)(1-\beta)}
$$

from which, we have

$$
N(\epsilon)=\left\lceil\log _{\beta} \frac{\delta \gamma(1-\beta)}{2\left(a_{2}+b_{2}\right)\left(a_{2}-a_{1}\right)}\right\rceil-1
$$

Since we have proved in the previous section that the optimal policy must have a switch curve structure, for any q, such that $q_{1} \geq N(\epsilon)$, the optimal policy is always to operate the system at point 2 . Thus, the switch curve has a limit.

The result implies that when both $q_{1}, q_{2}$ are large, the objective of maximizing the sum-rate is more important than balancing the queue lengths in order to minimize the average delay. Thus, in this scenario, operating at point 2 is optimal. When one queue ( $q_{1}$ in this paper) becomes close to empty, 
the objective of balancing the queue lengths becomes more important, and the operating point must be switched from point 2 to point 1 .

\section{NumericAl Results}

We consider a system where the arrival rates for the first and second user are $\lambda_{1}=0.4$ packets/unit time, $\lambda_{2}=0.3$ packets/unit time, respectively. We assume that the packet sizes are exponentially distributed i.i.d. random variables with unit mean. We assume that the underlying rate region is a general pentagon, where the normalized coordinates of the first corner point is $(0.3,0.5)$, and the normalized coordinates of the second corner point is $(0.7,0.3)$. We first obtain the optimal policy with $\beta=1$, which corresponds to the average delay minimization policy. We observe that the optimal policy has a switch structure. Then, we vary the value of $\beta$, and obtain the optimal policy for the corresponding discountedcost problem. These curves are shown in Fig. 6. We observe that for each curve, there is a limit on the dimension of $q_{1}$, and all of these curves are lower bounded by the curve with $\beta=1$. This can be explained in this way: as $\beta$ increases, the weight of future cost increases. Thus, balancing the queue lengths becomes progressively more important, and for some states, it overweighs maximizing the sum-rate at the current stage. Therefore, in this case, the set of states which operate at the first corner point enlarges.

\section{CONCLUSION}

In this paper, we investigated the delay minimization problem in a two-user multiple access channel, where the underlying rate region is approximated as a general pentagon. We assumed that the corner points of this pentagon have different sum-rates. We formulated the problem as an MDP, and proved that the delay-optimal policy operates at one of the two corner points, and has a switch structure. This implies that for some states, the optimal policy requires trading a portion of the sum-rate for balancing the queue lengths in order to minimize the average delay. We also proved that for the discounted-cost problem, the switch curve is bounded in one of the dimensions. This implies that the queues can be operated partially distributedly.

\section{APPENDIX}

\section{A. Proof of Lemma 3}

We prove the properties 1) through 4) of $T f$ by induction. If $f \in \mathcal{F}$, then obviously, $\mathbf{q}^{T} \mathbf{e}, f\left(A_{1} \mathbf{q}\right), f\left(A_{2} \mathbf{q}\right), f\left(D_{1} \mathbf{q}\right)$, $f\left(D_{2} \mathbf{q}\right)$ are in $\mathcal{F}$. Then, it suffices to show that $\min \left\{\left(b_{1}-\right.\right.$ $\left.\left.b_{2}\right) f\left(D_{2} \mathbf{q}\right)+\delta f(\mathbf{q}),\left(a_{2}-a_{1}\right) f\left(D_{1} \mathbf{q}\right)\right\}$ is also in $\mathcal{F}$. In order to simplify the notation, we define

$$
g(\mathbf{q})=\min \left\{\left(b_{1}-b_{2}\right) f\left(D_{2} \mathbf{q}\right)+\delta f(\mathbf{q}),\left(a_{2}-a_{1}\right) f\left(D_{1} \mathbf{q}\right)\right\}
$$

If $\left(b_{1}-b_{2}\right) f\left(D_{2} \mathbf{q}\right)+\delta f(\mathbf{q})<\left(a_{2}-a_{1}\right) f\left(D_{1} \mathbf{q}\right)$, then, the optimal operating point for state $\mathbf{q}$ is corner point 1 ; otherwise, the optimal operating point is corner point 2 . We will show that $g(\mathbf{q})$ also possesses the properties 1) through 4) of $f(\mathbf{q})$.

1) $g(\mathbf{q})$ is increasing in $q_{1}$ and $q_{2} .:$ It is straight forward to prove this property. Hence, we omit its proof.

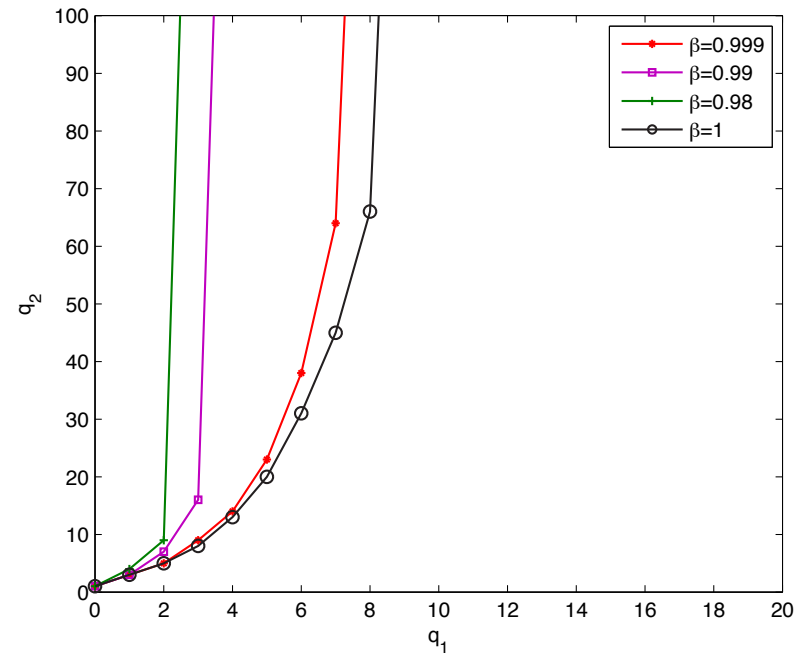

Fig. 6. The switch curves for the discounted-cost MDP.

2) $g(\mathbf{q}+\mathbf{x})-g(\mathbf{q})$ is increasing in $q_{1}$ and $q_{2}$ for any fixed $\mathrm{x}$. : For this property, we will prove that

$$
\begin{array}{r}
g\left(A_{1}^{2} \mathbf{q}\right)-g\left(A_{1} \mathbf{q}\right) \geq g\left(A_{1} \mathbf{q}\right)-g(\mathbf{q}) \\
g\left(A_{2}^{2} \mathbf{q}\right)-g\left(A_{2} \mathbf{q}\right) \geq g\left(A_{2} \mathbf{q}\right)-g(\mathbf{q}) \\
g\left(A_{1} A_{2} \mathbf{q}\right)-g\left(A_{2} \mathbf{q}\right) \geq g\left(A_{1} \mathbf{q}\right)-g(\mathbf{q})
\end{array}
$$

First, we evaluate function $g$ at points $\mathbf{q}, A_{1} \mathbf{q}, A_{1}^{2} \mathbf{q}$. If the optimal operating point for state $\mathbf{q}, A_{1} \mathbf{q}, A_{1}^{2} \mathbf{q}$ is corner point 1 , then, we have

$$
\begin{aligned}
g(\mathbf{q}) & =\left(b_{1}-b_{2}\right) f\left(D_{2} \mathbf{q}\right)+\delta f(\mathbf{q}) \\
g\left(A_{1} \mathbf{q}\right) & =\left(b_{1}-b_{2}\right) f\left(D_{2} A_{1} \mathbf{q}\right)+\delta f\left(A_{1} \mathbf{q}\right) \\
g\left(A_{1}^{2} \mathbf{q}\right) & =\left(b_{1}-b_{2}\right) f\left(D_{2} A_{1}^{2} \mathbf{q}\right)+\delta f\left(A_{1}^{2} \mathbf{q}\right)
\end{aligned}
$$

Comparing the difference of values between two adjacent states, we have

$$
\begin{aligned}
g\left(A_{1}^{2} \mathbf{q}\right)-g\left(A_{1} \mathbf{q}\right)= & \left(b_{1}-b_{2}\right)\left(f\left(D_{2} A_{1}^{2} \mathbf{q}\right)-f\left(D_{2} A_{1} \mathbf{q}\right)\right) \\
& +\delta\left(f\left(A_{1}^{2} \mathbf{q}\right)-f\left(A_{1} \mathbf{q}\right)\right) \\
\geq & \left(b_{1}-b_{2}\right)\left(f\left(D_{2} A_{1} \mathbf{q}\right)-f\left(D_{2} \mathbf{q}\right)\right) \\
& +\delta\left(f\left(A_{1} \mathbf{q}\right)-f\left(A_{1} \mathbf{q}\right)\right) \\
= & g\left(A_{1} \mathbf{q}\right)-g(\mathbf{q})
\end{aligned}
$$

where the inequality follows from the assumption that $f(\mathbf{q})$ is in $\mathcal{F}$. Similarly, if the optimal operating point for state $\mathbf{q}$, $A_{1} \mathbf{q}, A_{1}^{2} \mathbf{q}$ is corner point 2, i.e.,

$$
\begin{aligned}
g(\mathbf{q}) & =\left(a_{2}-a_{1}\right) f\left(D_{1} \mathbf{q}\right) \\
g\left(A_{1} \mathbf{q}\right) & =\left(a_{2}-a_{1}\right) f\left(D_{1} A_{1} \mathbf{q}\right) \\
g\left(A_{1}^{2} \mathbf{q}\right) & =\left(a_{2}-a_{1}\right) f\left(D_{1} A_{1}^{2} \mathbf{q}\right)
\end{aligned}
$$

we still have $g\left(A_{1}^{2} \mathbf{q}\right)-g\left(A_{1} \mathbf{q}\right) \geq g\left(A_{1} \mathbf{q}\right)-g(\mathbf{q})$.

If the optimal operating points for state $\mathbf{q}, A_{1} \mathbf{q}, A_{1}^{2} \mathbf{q}$ are corner points $1,2,2$, respectively, then, we have

$$
\begin{aligned}
g(\mathbf{q}) & =\left(b_{1}-b_{2}\right) f\left(D_{2} \mathbf{q}\right)+\delta f(\mathbf{q}) \\
g\left(A_{1} \mathbf{q}\right) & =\left(a_{2}-a_{1}\right) f\left(D_{1} A_{1} \mathbf{q}\right) \\
g\left(A_{1}^{2} \mathbf{q}\right) & =\left(a_{2}-a_{1}\right) f\left(D_{1} A_{1}^{2} \mathbf{q}\right)
\end{aligned}
$$




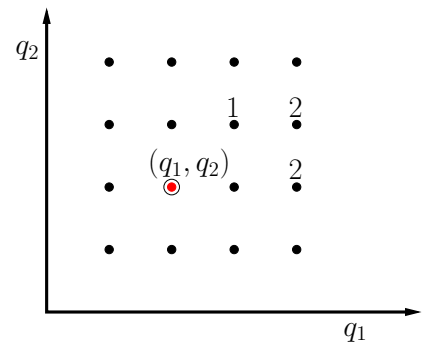

(a) Pattern 1

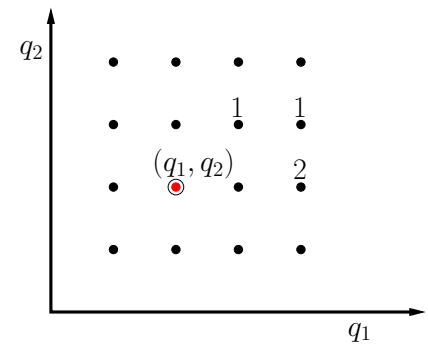

(b) Pattern 2

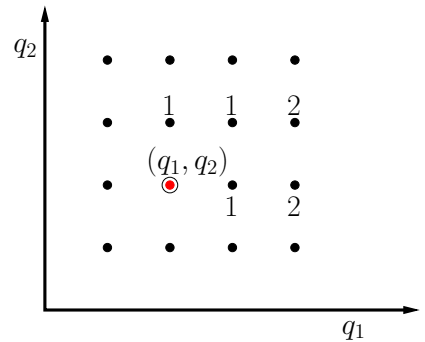

(a)

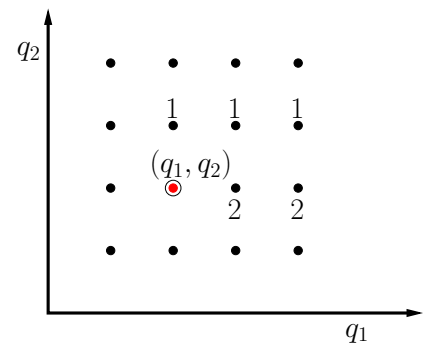

(b)

Fig. 7. Two special policy patterns.

Fig. 8. The optimal operating points at $A_{1}^{2} \mathbf{q}, A_{1} \mathbf{q}, A_{1}^{2} A_{2} \mathbf{q}, A_{1} A_{2} \mathbf{q}, A_{2} \mathbf{q}$.

and,

$$
\begin{aligned}
& g\left(A_{1} \mathbf{q}\right)-g(\mathbf{q}) \\
& =\left(a_{2}-a_{1}\right) f\left(D_{1} A_{1} \mathbf{q}\right)-\left(b_{1}-b_{2}\right) f\left(D_{2} \mathbf{q}\right)-\delta f(\mathbf{q}) \\
& =\left(b_{1}-b_{2}\right)\left(f(\mathbf{q})-f\left(D_{2} \mathbf{q}\right)\right) \\
& g\left(A_{1}^{2} \mathbf{q}\right)-g\left(A_{1} \mathbf{q}\right) \\
& \geq\left(a_{2}-a_{1}\right) f\left(A_{1} \mathbf{q}\right)-\left(b_{1}-b_{2}\right) f\left(D_{2} A_{1} \mathbf{q}\right)-\delta f\left(A_{1} \mathbf{q}\right) \\
& =\left(b_{1}-b_{2}\right)\left(f\left(A_{1} \mathbf{q}\right)-f\left(D_{2} A_{1} \mathbf{q}\right)\right)
\end{aligned}
$$

Therefore, based on the second property of function $f$, $g\left(A_{1}^{2} \mathbf{q}\right)-g\left(A_{1} \mathbf{q}\right) \geq g\left(A_{1} \mathbf{q}\right)-g(\mathbf{q})$ still holds.

Similarly, if the optimal operating points for state $\mathbf{q}, A_{1} \mathbf{q}$, $A_{1}^{2} \mathbf{q}$ are corner points $1,1,2$, respectively, we can prove in a similar way that property 2 ) still holds. The complete proof of this lemma can be found in [19].

Based on the assumption that $f \in \mathcal{F}$, if the optimal policy for any state $\mathbf{q}$ is to operate at corner point 2 , then, because of the third property of $f$, all the states $A_{1}^{n} \mathbf{q}, n>0$ should operate on point 2 also. In the analysis above, we discuss every possible policy at states $\mathbf{q}, A_{1} \mathbf{q}, A_{1}^{2} \mathbf{q}$. For all possible cases, we have shown that $g\left(A_{1}^{2} \mathbf{q}\right)-g\left(A_{1} \mathbf{q}\right) \geq g\left(A_{1} \mathbf{q}\right)-g(\mathbf{q})$. Following similar procedure, we can prove that $g\left(A_{2}^{2} \mathbf{q}\right)-$ $g\left(A_{2} \mathbf{q}\right) \geq g\left(A_{2} \mathbf{q}\right)-g(\mathbf{q})$, and $g\left(A_{1} A_{2} \mathbf{q}\right)-g\left(A_{2} \mathbf{q}\right) \geq$ $g\left(A_{1} \mathbf{q}\right)-g(\mathbf{q})$. In summary, we conclude that the property 2$)$ holds for $g(\mathbf{q})$.

3) $\left(a_{1}-a_{2}\right) g\left(D_{1} \mathbf{q}\right)+\left(b_{1}-b_{2}\right) g\left(D_{2} \mathbf{q}\right)+\delta g(\mathbf{q})$ is increasing in $q_{1} .:$ We need to show that

$$
\begin{aligned}
& \left(a_{1}-a_{2}\right) g\left(A_{1} A_{2} \mathbf{q}\right)+\left(b_{1}-b_{2}\right) g\left(A_{1}^{2} \mathbf{q}\right)+\delta g\left(A_{1}^{2} A_{2} \mathbf{q}\right) \\
& \geq\left(a_{1}-a_{2}\right) g\left(A_{2} \mathbf{q}\right)+\left(b_{1}-b_{2}\right) g\left(A_{1} \mathbf{q}\right)+\delta g\left(A_{1} A_{2} \mathbf{q}\right)
\end{aligned}
$$

We evaluate function $g$ at points $A_{1} A_{2} \mathbf{q}, A_{1}^{2} \mathbf{q}, A_{1}^{2} A_{2} \mathbf{q}, A_{2} \mathbf{q}$, $A_{1} \mathbf{q}$.

First, we note that if the optimal operating points for states $A_{1} A_{2} \mathbf{q}, A_{1}^{2} \mathbf{q}, A_{1}^{2} A_{2} \mathbf{q}$ are corner points $1,2,2$, respectively, as shown in Fig. 7(a),

$$
\begin{aligned}
g\left(A_{1} A_{2} \mathbf{q}\right) & =\left(b_{1}-b_{2}\right) f\left(D_{2} A_{1} A_{2} \mathbf{q}\right)+\delta f\left(A_{1} A_{2} \mathbf{q}\right) \\
g\left(A_{1}^{2} \mathbf{q}\right) & =\left(a_{2}-a_{1}\right) f\left(D_{1} A_{1}^{2} \mathbf{q}\right) \\
g\left(A_{1}^{2} A_{2} \mathbf{q}\right) & =\left(a_{2}-a_{1}\right) f\left(D_{1} A_{1}^{2} A_{2} \mathbf{q}\right)
\end{aligned}
$$

we have

$$
\begin{aligned}
& \left(a_{1}-a_{2}\right) g\left(A_{1} A_{2} \mathbf{q}\right)+\left(b_{1}-b_{2}\right) g\left(A_{1}^{2} \mathbf{q}\right)+\delta g\left(A_{1}^{2} A_{2} \mathbf{q}\right) \\
& =\left(a_{1}-a_{2}\right)\left(\left(b_{1}-b_{2}\right) f\left(D_{2} A_{1} A_{2} \mathbf{q}\right)+\delta f\left(A_{1} A_{2} \mathbf{q}\right)\right) \\
& +\left(b_{1}-b_{2}\right)\left(a_{2}-a_{1}\right) f\left(D_{1} A_{1}^{2} \mathbf{q}\right)+\delta\left(a_{2}-a_{1}\right) f\left(D_{1} A_{1}^{2} A_{2} \mathbf{q}\right) \\
& =0
\end{aligned}
$$

This is an important policy pattern, and we will use it often in the proof afterwards.

Another important policy patten is to operate at corner point $1,2,1$, for state $A_{1} A_{2} \mathbf{q}, A_{1}^{2} \mathbf{q}, A_{1}^{2} A_{2} \mathbf{q}$, respectively, as shown in Fig. 7(b). In this scenario, we observe that

$$
\begin{aligned}
& \left(a_{1}-a_{2}\right) g\left(A_{1} A_{2} \mathbf{q}\right)+\left(b_{1}-b_{2}\right) g\left(A_{1}^{2} \mathbf{q}\right)+\delta g\left(A_{1}^{2} A_{2} \mathbf{q}\right) \\
& =\left(a_{1}-a_{2}\right)\left(\left(b_{1}-b_{2}\right) f\left(D_{2} A_{1} A_{2} \mathbf{q}\right)+\delta f\left(A_{1} A_{2} \mathbf{q}\right)\right) \\
& \quad+\left(b_{1}-b_{2}\right)\left(a_{2}-a_{1}\right) f\left(D_{1} A_{1}^{2} \mathbf{q}\right) \\
& \quad+\delta\left(\left(b_{1}-b_{2}\right) f\left(D_{2} A_{1}^{2} A_{2} \mathbf{q}\right)+\delta f\left(A_{1}^{2} A_{2} \mathbf{q}\right)\right) \\
& =\delta\left(\left(a_{1}-a_{2}\right) f\left(A_{1} A_{2} \mathbf{q}\right)+\left(b_{1}-b_{2}\right) f\left(A_{1}^{2} \mathbf{q}\right)+\delta f\left(A_{1}^{2} A_{2} \mathbf{q}\right)\right)
\end{aligned}
$$

If the optimal operating points at $A_{1}^{2} \mathbf{q}, A_{1} \mathbf{q}, A_{1}^{2} A_{2} \mathbf{q}$, $A_{1} A_{2} \mathbf{q}, A_{2} \mathbf{q}$ are $2,1,2,1,1$, respectively, as shown in Fig. 8(a). Then, if we switch the operating point at state $A_{1} A_{2} \mathbf{q}$ from corner point 1 to 2 , the policy at point $A_{2} \mathbf{q}$, $A_{1} \mathbf{q}$, and $A_{1} A_{2} \mathbf{q}$ becomes the policy pattern discussed above, and we have

$$
\begin{aligned}
& \left(a_{1}-a_{2}\right) g\left(A_{2} \mathbf{q}\right)+\left(b_{1}-b_{2}\right) g\left(A_{1} \mathbf{q}\right)+\delta g\left(A_{1} A_{2} \mathbf{q}\right) \\
\leq & \left(a_{1}-a_{2}\right)\left(\left(b_{1}-b_{2}\right) f\left(D_{2} A_{2} \mathbf{q}\right)+\delta f\left(A_{2} \mathbf{q}\right)\right) \\
& +\left(b_{1}-b_{2}\right)\left(a_{2}-a_{1}\right) f\left(D_{1} A_{1} \mathbf{q}\right) \\
& +\delta\left(a_{2}-a_{1}\right) f\left(D_{1} A_{1} A_{2} \mathbf{q}\right) \\
= & 0 \\
= & \left(a_{1}-a_{2}\right) g\left(A_{1} A_{2} \mathbf{q}\right)+\left(b_{1}-b_{2}\right) g\left(A_{1}^{2} \mathbf{q}\right)+\delta g\left(A_{1}^{2} A_{2} \mathbf{q}\right)
\end{aligned}
$$

Similarly, if the optimal operating points at $A_{1}^{2} \mathbf{q}, A_{1} \mathbf{q}$, $A_{1}^{2} A_{2} \mathbf{q}, A_{1} A_{2} \mathbf{q}, A_{2} \mathbf{q}$ are $2,2,2,1,1$, or $2,2,2,2,1$, respectively, we can show that property 3 ) still holds.

If the optimal operating points at $A_{1}^{2} \mathbf{q}, A_{1} \mathbf{q}, A_{1}^{2} A_{2} \mathbf{q}$, $A_{1} A_{2} \mathbf{q}, A_{2} \mathbf{q}$ are $2,2,1,1,1$, as shown in Fig. 8(b), we have

$$
\begin{aligned}
& \left(a_{1}-a_{2}\right) g\left(A_{1} A_{2} \mathbf{q}\right)+\left(b_{1}-b_{2}\right) g\left(A_{1}^{2} \mathbf{q}\right)+\delta g\left(A_{1}^{2} A_{2} \mathbf{q}\right) \\
& =\delta\left(\left(a_{1}-a_{2}\right) f\left(A_{1} A_{2} \mathbf{q}\right)+\left(b_{1}-b_{2}\right) f\left(A_{1}^{2} \mathbf{q}\right)+\delta f\left(A_{1}^{2} A_{2} \mathbf{q}\right)\right) \\
& \geq \delta\left(\left(a_{1}-a_{2}\right) f\left(A_{2} \mathbf{q}\right)+\left(b_{1}-b_{2}\right) f\left(A_{1} \mathbf{q}\right)+\delta f\left(A_{1} A_{2} \mathbf{q}\right)\right) \\
& =\left(a_{1}-a_{2}\right) g\left(A_{2} \mathbf{q}\right)+\left(b_{1}-b_{2}\right) g\left(A_{1} \mathbf{q}\right)+\delta g\left(A_{1} A_{2} \mathbf{q}\right)
\end{aligned}
$$

where the inequality follows from the property 3 ) of function $f$, and the last inequality follows from the assumption that the policy at state $A_{2} \mathbf{q}, A_{1} \mathbf{q}, A_{1} A_{2} \mathbf{q}$ falls into the second policy pattern discussed above.

Similarly, if the optimal operating points at $A_{1}^{2} \mathbf{q}, A_{1} \mathbf{q}$, $A_{1}^{2} A_{2} \mathbf{q}, A_{1} A_{2} \mathbf{q}, A_{2} \mathbf{q}$ are $2,1,1,1,1$, the property still holds. In summary, for all possible cases, the function $g$ preserves the property 3 ) of function $f$. 


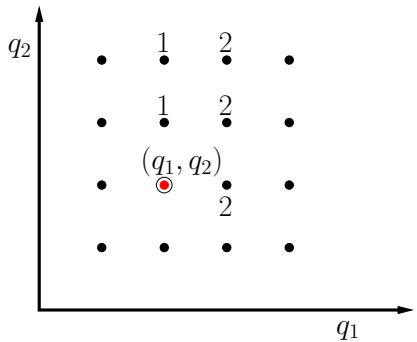

Fig. 9. The optimal operating points at $A_{1} \mathbf{q}, A_{1} A_{2} \mathbf{q}, A_{1} A_{2}^{2} \mathbf{q}, A_{2} \mathbf{q}, A_{2}^{2} \mathbf{q}$ are $2,2,2,1,1$, respectively.

4) $\left(a_{1}-a_{2}\right) g\left(D_{1} \mathbf{q}\right)+\left(b_{1}-b_{2}\right) g\left(D_{2} \mathbf{q}\right)+\delta g(\mathbf{q})$ is decreasing in $q_{2} .:$ We will evaluate $g$ at points $A_{1} \mathbf{q}, A_{1} A_{2} \mathbf{q}, A_{1} A_{2}^{2} \mathbf{q}$, $A_{2} \mathbf{q}, A_{2}^{2} \mathbf{q}$. If the optimal operating points are $2,2,2,2,2$, or $1,1,1,1,1$, respectively, it is straightforward to show that the property still holds. If the optimal operating points are $2,2,2,1,1$, as shown in Fig. 9, we note that the policy at these points is the first special policy patten discussed before, and

$$
\begin{aligned}
& \left(a_{1}-a_{2}\right) g\left(A_{2} \mathbf{q}\right)+\left(b_{1}-b_{2}\right) g\left(A_{1} \mathbf{q}\right)+\delta g\left(A_{1} A_{2} \mathbf{q}\right) \\
& =\left(a_{1}-a_{2}\right) g\left(A_{2}^{2} \mathbf{q}\right)+\left(b_{1}-b_{2}\right) g\left(A_{1} A_{2} \mathbf{q}\right)+\delta g\left(A_{1} A_{2}^{2} \mathbf{q}\right) \\
& =0
\end{aligned}
$$

Similarly, for cases where the optimal operating points are $2,2,1,1,1$, or $2,2,2,2,1$, or $2,2,1,2,1$, or $2,2,1,1,1$, the property 4) still holds for $g$. In summary, for all possible cases, we have proven that properties 1) through 4) hold for $g$, thus, if $f$ is in $\mathcal{F}$, then $T f$ is in $\mathcal{F}$.

\section{REFERENCES}

[1] J. Yang and S. Ulukus, "Delay minimization with a general pentagon rate region," IEEE International Symposium on Information Theory, June 2010

[2] A. Ephremides and B. Hajek, "Information theory and communication networks: An unconsummated union," IEEE Trans. Inf. Theory, vol. 44, pp. 2416-2434, Oct 1998.

[3] E. Yeh, "Delay-optimal rate allocation in multiaccess communications: A cross-layer view," IEEE Workshop on Multimedia Signal Processing, pp. 404- 407, Dec 2002.

[4] E. Yeh and A. Cohen, "Throughput and delay optimal resource allocation in multiaccess fading channels," IEEE International Symposium on Information Theory, p. 245, Jun/Jul 2003.

[5] - -, "Delay optimal rate allocation in multiaccess fading communications," Allerton Conference on Communication, Control, and Computing, pp. 140-149, Sep/Oct 2004

[6] N. Ehsan and T. Javidi, "Delay optimal transmission policy in a wireless multi-access channel," IEEE Trans. Inf. Theory, no. 8, pp. 3745-3751, Aug 2008.

[7] E. Yeh, "Minimum delay multi-access communication for general packet length distributions," 42nd Annual Allerton Conference on Communication, Control, and Computing, pp. 1536-1545, Sep/Oct 2004.

[8] S. Musy, "Delay and coding in multiple-user communications," Ph.D. dissertation, EPFL, 2007.

[9] J. Yang and S. Ulukus, "Delay minimization in multiple access channels," IEEE International Symposium on Information Theory, June/July 2009.

[10] T. M. Cover and J. A. Thomas, Elements of Information Theory. New York: John Wiley and Sons, Inc, 1991.

[11] D. Tse and S. Hanly, "Multiaccess fading channels - Part I: Polymatroid structure, optimal resource allocation and throughput capacities," IEEE Trans. Inf. Theory, vol. 7, pp. 2796-2815, 1998.
[12] W. Yu, W. Rhee, S. Boyd, and J. Cioffi, "Iterative water-filling for Gaussian vector multiple access channels," IEEE Trans. Inf. Theory, vol. 50, no. 1, pp. 145-151, Jan 2004.

[13] A. Sendonaris, E. Erkip, and B. Aazhang, "User cooperation diversityPart I: System description," IEEE Trans. Commun., vol. 51, no. 11, pp. 1927-1938, Nov 2003

[14] O. Kaya and S. Ulukus, "Power control for fading cooperative multiple access channels," IEEE Trans. Wireless Commun., vol. 6, no. 8, pp. 2915-2923, August 2007.

[15] V. G. Kulkarni, Modeling and Analysis of Stochastic Systems. Chapman and Hall/CRC, 1995.

[16] J. Walrand, An Introduction to Queueing Networks. Prentice Hall, 1988

[17] W. Lin and P. R. Kumar, "Optimal control of a queueing system with two heterogenous servers," IEEE Trans. Autom. Control, vol. 29, pp. 696-703, Aug 1984.

[18] B. Hajek, "Optimal control of two interacting service stations," IEEE Trans. Autom. Control, vol. 29, no. 6, pp. 491-499, June 1984.

[19] J. Yang, "Delay minimization in energy constrained wireless communications," Ph.D. dissertation, University of Maryland, College Park, 2010.

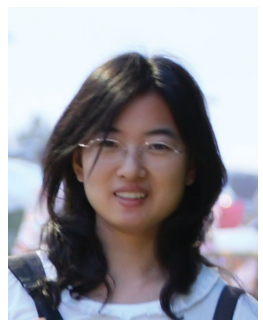

Jing Yang received the B.S. degree in electronic engineering and information science from University of Science and Technology of China, Hefei, China in 2004, and the M.S. and Ph.D. degrees in electrical and computer engineering from the University of Maryland, College Park in 2010. Since October 2010, she has been a research associate in the department of electrical and computer engineering at the University of Wisconsin-Madison. Her research interests are in wireless communication theory and networking, multi-user information theory, queueing theory, optimization in wireless networks, and statistical signal processing.

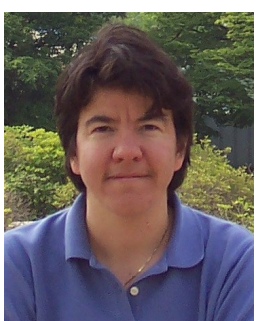

Sennur Ulukus received the B.S. and M.S. degrees in electrical and electronics engineering from Bilkent University, Ankara, Turkey, in 1991 and 1993, respectively, and the Ph.D. degree in electrical and computer engineering from Rutgers University, $\mathrm{NJ}$, in 1998

During her Ph.D. studies, she was with the Wireless Information Network Laboratory (WINLAB), Rutgers University. From 1998 to 2001, she was a Senior Technical Staff Member at AT\&T LabsResearch in NJ. In 2001, she joined the University of Maryland at College Park, where she is currently an Associate Professor in the Department of Electrical and Computer Engineering, with a joint appointment at the Institute for Systems Research (ISR). Her research interests are in wireless communication theory and networking, network information theory for wireless networks, signal processing for wireless communications and security for multi-user wireless communications.

Dr. Ulukus is a recipient of the 2005 NSF CAREER Award, and a co-recipient of the 2003 IEEE Marconi Prize Paper Award in Wireless Communications. She serves/served as an Associate Editor for the IEEE Transactions on Information Theory since 2007, as an Associate Editor for the IEEE Transactions on Communications between 2003-2007, as a Guest Editor for the IEEE Transactions on Information Theory for the special issue on interference networks, as a Guest Editor for the IEEE Journal on Selected Areas in Communications for the special issue on multiuser detection for advanced communication systems and networks, as the co-chair of the Communication Theory Symposium at the 2007 IEEE Global Telecommunications Conference, as the co-chair of the Medium Access Control (MAC) Track at the 2008 IEEE Wireless Communications and Networking Conference, as the co-chair of the Wireless Communications Symposium at the 2010 IEEE International Conference on Communications, as the co-chair of the 2011 Communication Theory Workshop, as the co-chair of the PhysicalLayer Security Workshop at the 2011 IEEE International Conference on Communications, and as the Secretary of the IEEE Communication Theory Technical Committee (CTTC) in 2007-2009. 